\title{
A Middle School Campus Case Analysis with Dynamic Post- evaluation Strategy of Space Syntax
}

\author{
Kexin Guo ${ }^{1}$, Jining Pang ${ }^{2}$, Guoqing Peng ${ }^{2 *}$, Xuan $\mathrm{Qu}^{2}$, Chunmei Pang ${ }^{2}$, Qian Wan ${ }^{2.3}$ \\ ${ }^{1}$ School of Information Engineering, China University of Geosciences (Beijing), Beijing 100083, China \\ ${ }^{2}$ Hualan Design and Consulting Group Company Ltd., Nanning 530011, China \\ ${ }^{3}$ School of Architecture and Transportation Engineering, Guilin University of Electronic Technology, Guilin 541004, China
}

\begin{abstract}
Due to the lack of technology, the post-evaluation system of spatial syntax has the limitations of inexperience, 3D vision and spatial problems prediction. The paper focuses on: (1) combined with the layout evaluation case of Nanning No.2 Middle School, the application of dynamic post-evaluation system in the evaluation process is refined, and the rationality of the system is verified; (2) combined with sDNA and GIS, a isovist model is built to expand the spatial syntax in 3D; (3) the simulation model is integrated into the post-evaluation system to solve the problem of dynamic feedback space, which enriches the dynamic post-evaluation system and improves the efficiency of campus layout.
\end{abstract}

\section{INTRODUCTION}

As a means of spatial logic analysis, spatial syntax provides the possibility of quantitative evaluation of campus layout through the relation between spatial organization and spatial function. The space syntax analysis with Depthmap has great limitations in campus layout evaluation [1-2]. Researches mainly described the parameter values of each element, the conclusion cannot provide guidance for practical application. Although, a quantitative, visual and procedural dynamic post-evaluation system was proposed, which refines the application of spatial syntax in campus layout evaluation, there is no verification analysis combined with specific cases, and there is no solution for $3 \mathrm{D}$ campus layout evaluation and dynamic simulation technology.

The application of space syntax in 3D scenes is limited. At present, scholars have made explorations on improving its 3D applicability, and most of them focus on the method of isovist analysis [3]. Yongwang Zhou [4] used the average slope as the index of intervisibility to get the $3 \mathrm{D}$ isovist by Kruger interpolation, Jingwen Wang[5] uses the weighted integration degree to expand the calculation. In this study, sDNA model was used. sDNA improves the rationality of axis model on the basis of spatial syntax, and relies on ArcGIS to improve its computing efficiency [6]. It not only gives full play to the advantages of GIS spatial analysis, enriches spatial evaluation methods, but also can assist 3D planning and design to a certain extent.

Dynamic evaluation is an important part of campus layout planning. On the basis of students' behavior law, according to gathering behavior in campus, the Anylogic discrete event model is established to verify the feasibility of the system and optimize the space.
This study applied a methodology from $A$ dynamic postevaluation analysis strategy based on Space syntax for optimizing middle school campus layout[7] (submitted for publish) to investigate Nanning No.2 Middle School, combined with sDNA, GIS, Anylogic technology to solve the difficult problems of space syntax in the evaluation of campus layout, and compared with the traditional space syntax to verify the advantages of the method, and further enriched the application experience in the dynamic post-evaluation system of middle school campus, provided technical guidance for the evaluation of middle school campus layout.

\section{CASE}

\subsection{First stage: spatial cognition of Nanning No.2 Middle School}

Nanning No.2 Middle School is the best middle school of Guangxi Zhuang Autonomous Region. It has obvious terrain elevation difference and typical campus layout.

\subsection{Second / Third stage: syntax model establishment and planning evaluation Position}

\subsubsection{Pedestrian road system}

Space syntax calculates the parameters of axis model based on Depthmap, explained them directly, without discussing the matching with space function. Combined with the case of Nanning No.2 Middle School, the paper established the spatial syntax axis model, and the analysis results are shown in Figure 1, and sDNA

\footnotetext{
*Corresponding author: 243564846@qq.com
} 
analysis was carried out to establish line segment models at three scales: micro (5 min travel radius), meso (10 min travel radius) and macro to evaluate the accessibility and carrying capacity under characteristic travel mode (Figure 2).
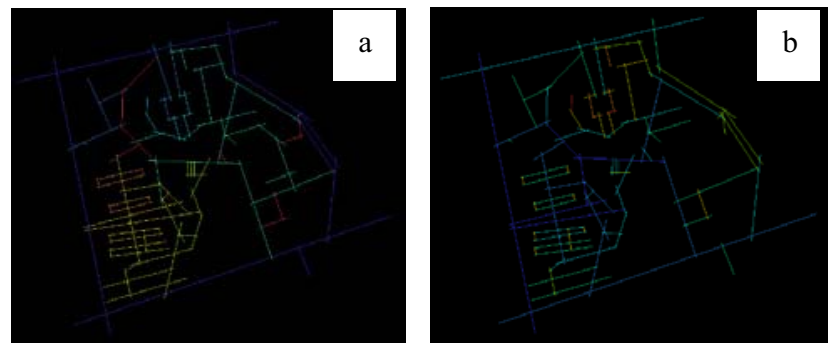

Figure. 1. Space syntax axis map analysis diagram (a) choice (b) integration

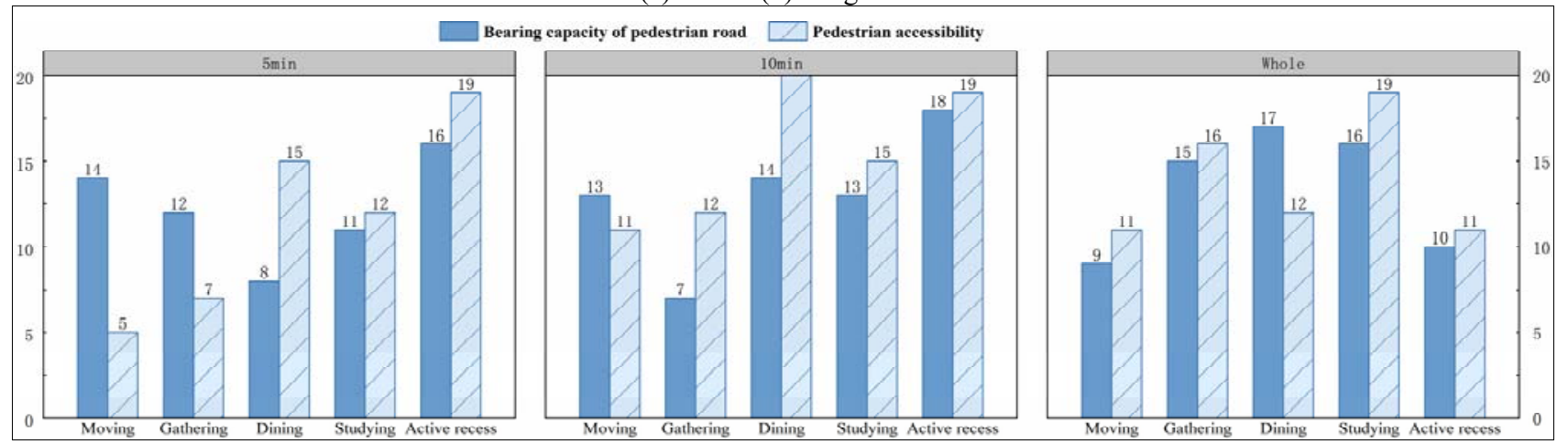

Figure 2. parameter diagram of segment model

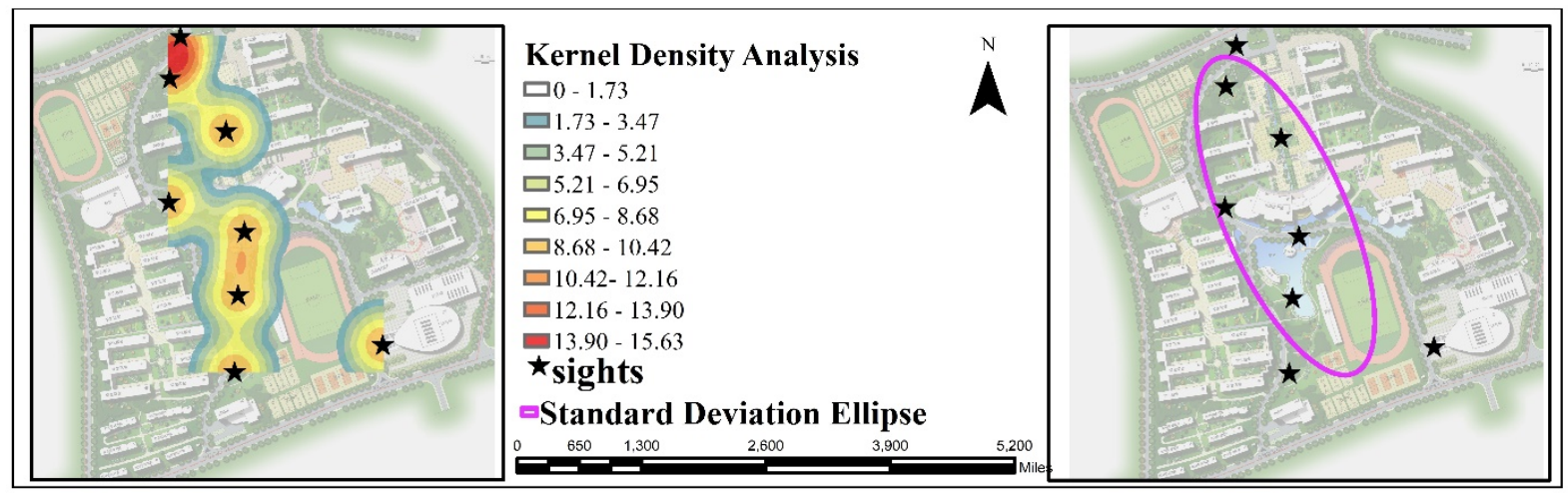

Figure 3. The landscape analysis map of Nanning No.2 Middle School

Because of the requirement of high accessibility and high road carrying capacity of the road system, it is typical to take gathering behavior as an example to evaluate the rationality of the method. The road space from the teaching area / dormitory area to the assembly site ( $400 \mathrm{~m}$ playground) is evaluated by calculating the choice and integration of the line model at each scale. From the results of parameter analysis, the road carrying capacity and accessibility are medium in 5-minute travel scale, low in 10-minute travel scale, high in main roads in teaching area and dormitory area, and low in branch roads connecting $400 \mathrm{~m}$ playground. Generally speaking, the carrying capacity of the pedestrian road is low, the accessibility is not strong, and it cannot support largescale activities, which is bound to cause congestion and limit travel, so space adjustment and improvement are needed.
The analysis logic of pedestrian road system evaluation under other typical travel behaviors is similar to the above cases. Compared with spatial syntax, the segment model is more sensitive to the topology of the road network and has less space errors. It highlights the travel rules and travel characteristics of space users. The evaluation parameters of sDNA model road system have good correspondence with its space. At the same time, it can better explore the matching degree of space status and space function from the perspective of space users.

\subsubsection{Green landscape system}

The eight sceneries of Nanning No.2 Middle School are the memory sceneries of it. Through kernel density analysis and standard deviation ellipse analysis, this paper evaluates the distribution density and distribution geometry of eight scenic nodes in Nanning No.2 Middle 
School, verifies whether the distribution trend meets the needs of landscape axis design, and further seeks and avoids isolated landscape.

The landscape analysis map of Nanning No.2 Middle School is shown in Figure 4. From the perspective of distribution density, the eight sceneries of the campus are densely distributed, and the landscape system is relatively continuous, running through the whole teaching and leisure area. From the perspective of distribution geometry, the eight sceneries of the campus take the north-south direction as the distribution axis, showing a north-south zonal area. But Forever Song, Changong Yueying and Yangzhi Pavilion are far away from the core area, so landscape corridor should be added to strengthen the connection between campus landscapes.

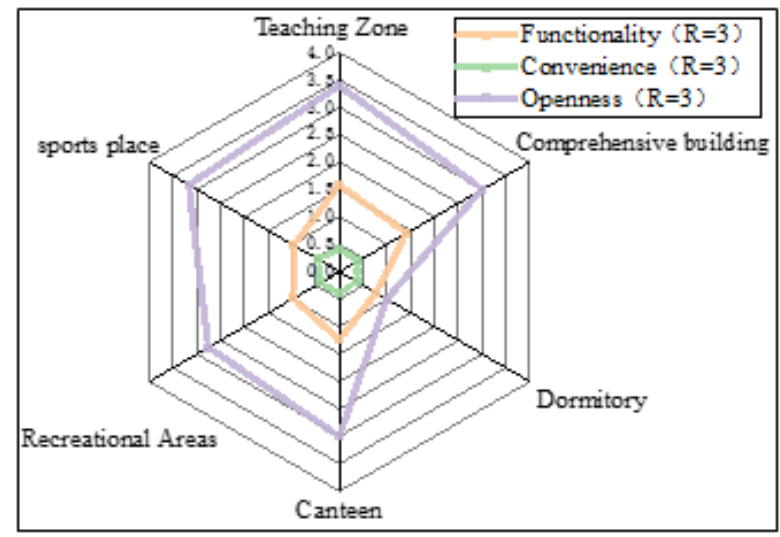

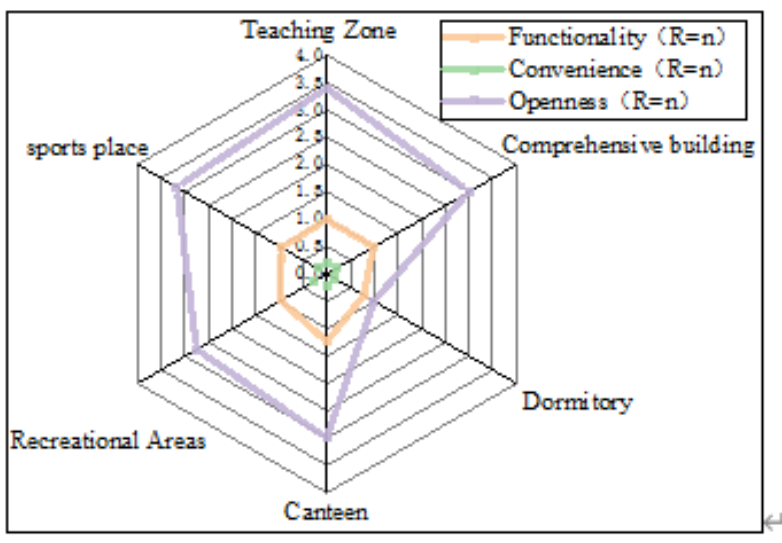

Figure 4. Parameter analysis chart of sDNA convex model

\subsubsection{Open space system}

The authors established local and global spatial syntactic convex models for the six typical functional areas in the middle school campus, get the specific parameters (Figure 4), and evaluate the space according to its spatial functionality (Table 2).

\begin{tabular}{|c|c|c|}
\hline Space & Function \\
\hline Sports place & High convenience & Highly open and \\
Cunctional/convenience & \\
\hline Recess activities & Low openness/medium convenience & \\
\hline Teaching Zone & High functionality /medium openness & \\
\hline Canteen & High convenience /medium openness & \\
\hline Dormitory & Low openness and low functionality & \\
\hline
\end{tabular}

In addition, line of sight accessibility evaluation is also an important part. In the conventional research, mainly uses the perspective analysis of spatial syntax to evaluate the accessibility of spatial line of sight. which is verified the rationality and feasibility of the application of the perspective analysis model. This study established a visibility graph for the campus of Nanning No.2 Middle School (Figure 5).

The transition from red to blue indicates a decrease in visual field control. Through the horizon analysis, we can see that the sight control of the courtyard area of the teaching building is poor, which is contrary to the field research results. Since Nanning No. 2 Middle School has unique $3 \mathrm{D}$ terrain features That requires to consider the influence of 3D elements on the observation field of vision. Therefore, this study integrates 3D observer points analysis of GIS 3D analysis, and tries to analyze

\section{Characteristics}

High convenience, reasonable layout

The convenience, functionality and openness are medium, but the core status is not reflected

The openness is medium, and the concealment needs to be improved Proper function and reasonable layout

Convenience, openness and functionality are too high, and space resources are wasted

Low openness, high convenience and reasonable layout

the view in 3D scene. Firstly, establish a 3D terrain plane based on elevation interpolation which corrected and adjusted with the help of field survey data ; secondly, built campus 3D scene, and set the observer points are in typical building spaces such as teaching building, comprehensive building, etc., and the visual field under specific viewpoints is quantitatively evaluated; finally, realized the $3 \mathrm{D}$ visual field analysis and evaluation map on ArcScene (Figure 6), in which the red is visible. It can be seen that the view analysis considering the influence of $3 \mathrm{D}$ terrain is more in line with the actual situation of the campus, and is more reasonable than the traditional space syntax view analysis. 


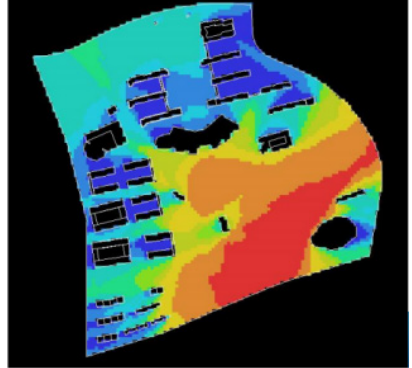

Figure 5. Isovist analysis of space syntax

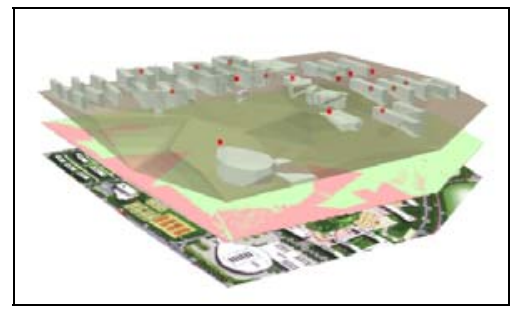

Figure 6. Viewpoint analysis of GIS

\subsection{The fourth stage: simulation verification and space optimization}

Through the above model establishment and quantitative evaluation of the matching of space and its functions, the paper digs out the hidden space problems in Nanning No.2 Middle School, such as road bearing capacity and accessibility cannot fully meet the needs of road system

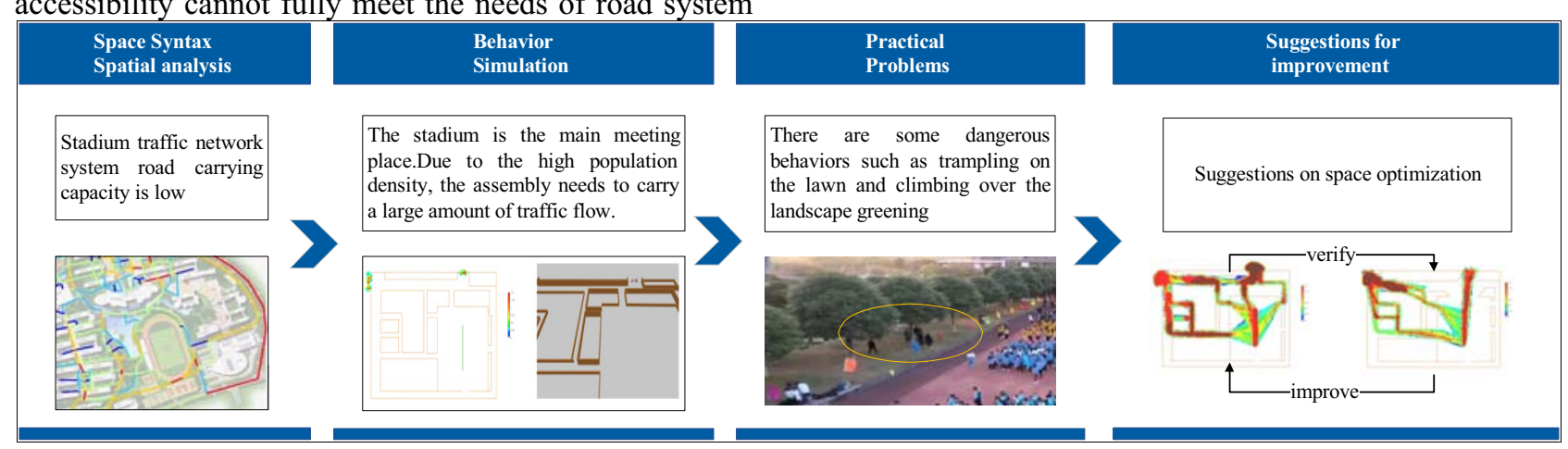

Figure 7. Simulation verification and space optimization analysis logic

\section{CONCLUSIONS}

This study combines sDNA, GIS and social force simulation technology to provide technical support for the evaluation of middle school campus layout. Taking Nanning No.2 Middle School as an example, this paper established a 3D isovist model combined with GIS and SDNA, and compares it with the isovist analysis results of spatial syntax, effectively improved the 3D expansibility of spatial syntax; combined with the dynamic post-evaluation system, this paper refines the application of spatial syntax in the evaluation of middle school campus layout, and verifies its rationality and logical integrity through case analysis. The simulation model is integrated into the post-evaluation of campus layout to dynamically feedback space problems and supporting $400 \mathrm{~m}$ playground as a large assembly place. This paper constructed the complete behavior logic, built the social force model. Combined with the collected and analyzed data, set the pedestrian flow distribution proportion of the two main entrances and exits in the teaching area and stop after 12 minutes, so as to get the result from the teaching area to the campus. The crowd density map further explains that the mismatch between spatial structure and spatial function is the cause of spatial problems, and also verifies the rationality of spatial syntactic space evaluation.

According to the spatial evaluation of the spatial syntax model and the analysis of the dynamic simulation model, the imperfect road system on the south side of the complex building cannot hold the crowd gathering effect of the $400 \mathrm{~m}$ playground, which is the reason for the behaviors such as wasting time on traveling unnecessary distance and crossing the green belt in Nanning No.2 Middle school. Therefore, this study puts forward the corresponding adjustment suggestions. For example: (1) open the north side of the semi-enclosed green, to ease the flow of people, and enhance people's sense of participation in the landscape. (2) The width of the road at the southwest entrance should be widened to adjust the space. The optimized passenger flow density map can be seen that the passenger flow density of each main entrance of the playground has decreased significantly, and the congestion on the north side has been effectively alleviated. The analysis logic is shown in Figure 7. 


\section{References}

1. Xu J, Wang B, Research of Chicago University Campus Deep Construction Based on Space Syntax Analysis. Huazhong Architecture, 10: 137-139 (2010).

2. Zhang Y, A Cognitive Study of Campus Spatial Morphology Based on Spatial Syntax-Take Wuhan University Campus as an Example. Architecture\& Culture, 07: 131-132 (2020).

3. Zhang X, Cheng Z, Bai Y, (2014), Review and Prospect of Space Syntax. Geography and GeoInformation Science, 08:75-80.

4. Zhou Y, Tian C, Li M, Urban visibility analysis using 3D space syntax. Computer Engineering and Applications, 1: 240-241(2014).

5. Wang J, Zhu Q, Mao Q, Discussion about the Three-dimensional Extension of Space Syntax. Huazhong Architecture, 08: 75-80 (2007).

6. Ma F, Spatial equity analysis of urban green space based on spatial design network analysis (sDNA): A case study of central Jinan, China, 60 (2020).

7. Guo K, et al., A dynamic post-evaluation analysis strategy based on Space syntax for optimizing middle school campus layout, 2021, submitted for public. 